\title{
Rare Simultaneous Left and Right-Sided Native Valve Infective Endocarditis Caused by Rare Bacterium
}

\author{
Jian-Hong Pan, ${ }^{1}$ MD
}

\begin{abstract}
Summary
Simultaneous left and right-sided native valve infective endocarditis (IE) is rare. Staphylococcus aureus was the predominant organism for bilateral IE. Shunt diseases are common risk factors of both-sided IE. Streptococcus anginosus (S. anginosus) is usually associated with pyogenic infections, but rarely a cause of IE. Here we present an extremely rare case of simultaneous left and right-sided native valve IE affecting the mitral and tricuspid valves caused by S. anginosus in an adult patient that has not been reported in the literature previously, particularly without the most frequent predisposing factors of IE. A 66-year-old man was admitted due to generalized fatigue, chills, malaise, and intermittent fevers for 1 year. A grade III-IV/VI systolic murmur at the mitral area and a III/VI systolic murmur at the tricuspid area were noted on physical examination. Laboratory evaluation revealed an elevated erythrocyte sedimentation rate and C-reactive protein level, and high fasting blood glucose. Blood culture was positive for S. anginosus. Echocardiography revealed vegetations in both sides of the heart: a large mitral valve vegetation with severe mitral regurgitation, as well as another vegetation on the tricuspid valve with moderate regurgitation. The case highlights a rare pathogen of both-sided IE, a rare presentation of S. anginosus infection, and several points worthy of note in echocardiography of IE.
\end{abstract}

(Int Heart J 2019; 60: 474-476)

Key words: Streptococcus anginosus, TTE, TEE

I nfective endocarditis (IE) is a microbial infection of heart valves and its endothelial lining. Despite undeniable improvements in medical and surgical treatment over the last decades, IE remains a life-threatening disease.") IE most frequently involves left-sided cardiac structures. Simultaneous left and right-sided native valve IE is uncommon. Staphylococcus aureus (S. aureus) is the predominant organism for both-sided IE. IE occurs predominantly in patients with valvular heart disease, a prosthetic valve, intravenous drug abuse, cardiac device insertion, or congenital heart disease. Intracardiac or extracardiac shunts are common risk factors of both-sided IE. Streptococcus anginosus (S. anginosus) belongs to the Streptococcus milleri group which is more prone to causing purulent infections with abscess formation, but is rarely reported as the cause of IE. Both-sided native valve IE caused by $\mathrm{S}$. anginosus has not been reported in the literature previously, particularly in patients who lack the common risk factors of IE. An extremely rare case of simultaneous left and right-sided native valve IE caused by a rare pathogen of IE, S. anginosus, without frequent risk factors of IE, is reported herein.

\section{Case Report}

A 66-year-old man was admitted to our hospital with generalized fatigue, chills, malaise, intermittent fevers for 1 year (maximum body temperature at $39.2^{\circ} \mathrm{C}$ ), incidental cough and white phlegm, without chest distress, dyspnea, palpitations, and weight loss. The patient had a history of diabetes mellitus for more than 1 year prior and was treated with subcutaneous insulin injections. He had no prior history of valvular heart disease, prosthetic valve, intravenous drug abuse, cardiac device insertion, or congenital heart disease. The patient always improved after shortterm antibiotic and symptomatic treatment but suffered a relapse later.

On physical examination, he had an axillary temperature of $36.7^{\circ} \mathrm{C}$, blood pressure of $130 / 70 \mathrm{mmHg}$, heart rate of $103 \mathrm{bpm}$, and respiratory rate of 20/minute. The patient was in a good general condition upon admission, without concomitant heart failure or hemodynamic instability. While in hospital, he was febrile up to $37.6^{\circ} \mathrm{C}$. A grade III-IV/VI systolic murmur at the mitral area and a III/VI systolic murmur at the tricuspid area were noted. No extremity petechiae were detected.

Laboratory evaluation revealed a leukocyte count of $7.06 \times 109 / \mathrm{L}$ with $83.3 \%$ neutrophils, an elevated erythrocyte sedimentation rate of $38 \mathrm{~mm} / \mathrm{h}$ (normal range, $0-15$ $\mathrm{mm} /$ hour), C-reactive protein of $33.05 \mathrm{mg} / \mathrm{L}$ (normal range, $0.068-8.2 \mathrm{mg} / \mathrm{L}$ ), high fasting blood glucose of $7.86 \mathrm{mmol} / \mathrm{L}$, and brain natriuretic peptide of $112 \mathrm{pg} / \mathrm{mL}$

From the ${ }^{1}$ Department of Internal Medicine, Tianjin NanKai Hospital, Tianjin, China.

Address for correspondence: Jian-Hong Pan, MD, Department of Internal Medicine, Tianjin NanKai Hospital, Tianjin, China, No.6 Changjiang Road, Nankai District, Tanjin 300100, China. E-mail: nankaiyiyuanxgn@126.com

Received for publication June 2, 2018. Revised and accepted July 31, 2018

Released in advance online on J-STAGE January 10, 2019.

doi: 10.1536/ihj.18-347

All rights reserved by the International Heart Journal Association. 

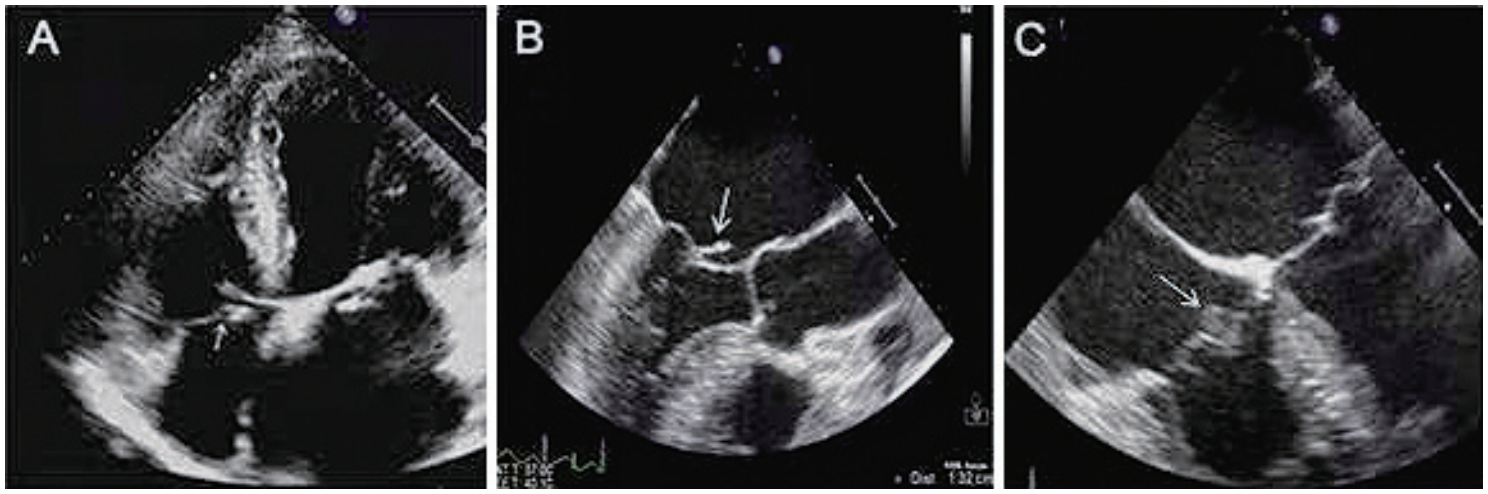

Figure. A: TTE on admission revealed the anterior leaflet of the tricuspid valve was prolapsed (arrow). B: TEE long-axis view of the aortic valve showed the vegetation attached to the anterior leaflet of the mitral valve (arrow). C: TEE 4-chamber view showed a vegetation attached to the anterior leaflet of the tricuspid valve (arrow), which was mistaken as the prolapsed anterior leaflet of the tricuspid valve in TTE.

(normal range, $0-100 \mathrm{pg} / \mathrm{mL}$ ). Blood culture was positive for S. anginosus, which was sensitive to ceftriaxone (MIC $0.25 \mu \mathrm{g} / \mathrm{mL}$ ), cefotaxime (MIC $0.25 \mu \mathrm{g} / \mathrm{mL}$ ), vancomycin (MIC $1 \mu \mathrm{g} / \mathrm{mL}$ ), and levofloxacin (MIC $1 \mu \mathrm{g} / \mathrm{mL}$ ).

Chest computed tomography (CT) was performed and showed multiple bilateral pulmonary nodules. Splenomegaly, kidney stones, and a cyst in the right kidney were observed with abdominal ultrasound. Electrocardiography showed sinus tachycardia. Echocardiography was performed. Transthoracic echocardiography (TTE) was done initially. Typical presentation of vegetation was observed; a $15 \mathrm{~mm} \times 5 \mathrm{~mm}$ oscillating mass attached to the anterior leaflet of the mitral valve, which moved independently to the valve with severe mitral regurgitation. Additionally, TTE revealed the anterior leaflet of the tricuspid valve was prolapsed with moderate regurgitation (Figure A). Transesophageal echocardiography (TEE) was also performed the next day. Another tricuspid valve vegetation was observed apart from the mitral valve vegetation (Figure B); a $9 \mathrm{~mm} \times 3 \mathrm{~mm}$ mobile mass attached to the anterior leaflet of the tricuspid valve (Figure C) with moderate tricuspid regurgitation.

An empiric antimicrobial had been initiated prior to the blood culture result. The patient's symptoms improved gradually. The diagnosis of IE was correctly made according to the modified Duke criteria. ${ }^{2)}$ Cardiovascular surgeons were consulted and they recommended surgical intervention. The patient was transferred for further cardiothoracic surgical management. Vegetations attached to the mitral and tricuspid valves were confirmed in surgery. The patient underwent mitral valve replacement and tricuspid annuloplasty. He recovered well and was discharged.

\section{Discussion}

We present here a rare case that has not been reported in the literature before. The case highlights a rare pathogen of both-sided IE, a rare presentation of S. anginosus infection, and several points worthy of note in echocardiography of IE.

IE most frequently involves left-sided cardiac struc- tures. The incidence of right-sided IE (RIE) is much lower, accounting for 5\%-10\% of all cases of IE. ${ }^{3)}$ Reports of both-sided IE are scarce. Only two cases of both-sided native valve IE were retrieved in the literature. ${ }^{4,5)}$ Both patients had a history of intravenous drug abuse - a common risk factor of IE. One case was caused by S. aureus, and Gram-positive cocci (in all probability S. aureus) were seen in the vegetations of the other case. The case we present was caused by S. anginosus and he was not a drug addict. This might be the first case of both-sided native valve IE caused by $\mathrm{S}$. anginosus without common predisposing factors of IE.

IE occurs predominantly in patients with valvular heart disease, a prosthetic valve, intravenous drug abuse, medical device implantation, or congenital heart disease. RIE occurs predominantly in intravenous drug users. ${ }^{6}$ Intracardiac or extracardiac shunt diseases (e.g., ventricular septal defects, patent ductus arteriosus) are common risk factors of both-sided IE. ${ }^{7}$ The shunting can result in the development of IE from single side to both sides of the heart involvement. Nevertheless, this patient did not have RIE.

S. aureus was the most frequent pathogen of RIE, ${ }^{8)}$ and both-sided IE. In our case, the isolated pathogen was $\mathrm{S}$. anginosus, which is a member of the S. milleri group. The S. milleri group consists of 3 distinct species: S. constellatus, S. anginosus, and S. intermedius. ${ }^{9)}$ S. milleri is considered a part of the resident flora of the oral cavity and upper respiratory tract. ${ }^{10)}$ Although Streptococci have been well known as a causative agent of IE, some groups (e.g. S. milleri) are rarely reported as being associated with IE. The S. milleri group is more prone to causing purulent infections with abscess formation, ${ }^{11)}$ but rarely causes IE. ${ }^{12)}$ Both-sided native valve IE caused by S. anginosus, as seen in our case, has not been previously reported in the literature. Hence, the isolation of S. anginosus from the blood of this patient was unexpected.

The pathogenesis of both-sided native valve IE in this patient is not clear due to his lack of common predisposing factors of IE. It is worth noting that he was healthy in the past but had the coexisting condition of diabetes for over 1 year. Diabetes might be a risk factor and 
play a role in the development of IE. In addition, nonstandard antibiotic treatment before admission could be responsible for the long disease duration. The persistent bacteremia made it possible for both sides of the heart to be involved. However, this is only speculation. Pulmonary arteriovenous shunt is another shunt disease which is not as common as those mentioned above. Although no cyanosis and no dyspnea were present in this patient, a pulmonary arteriovenous fistula could not be excluded. It might be the key enabler of both-sides involvement, especially in the absence of common risk factors. Contrast echocardiography could demonstrate this extracardiac right-to-left shunt and should be performed.

Echocardiography is pivotal to the diagnosis and management of patients with IE. Vegetations are the hallmark lesions of IE. It is important to identify vegetations early for optimal treatment. TTE, as the best noninvasive visualization technique, can detect the majority of IE. TEE is significantly more sensitive than TTE for the detection of vegetations and abscesses of IE due to its higher spatial resolution and multi-angle visualization of the structures. It is worth mentioning that the results of echocardiography are influenced by many factors. For example, since RIE is relatively rare, vegetations attached to the right sided valves are likely missed. Therefore, the experience and skill of the examiner can influence the examination results. Furthermore, image quality, echogenicity, vegetation sizes and location, previous valvular disease, or valvular prosthesis could affect the outcome. Meanwhile, the examiner must take care to avoid pitfalls in echocardiography otherwise a misdiagnosis might be made. Sometimes it is difficult to differentiate vegetation from a prolapsed cusp, as our case described. The tricuspid valve vegetation was mistaken as a prolapsed anterior leaflet of the tricuspid valve in the first echocardiography examination. In addition, vegetations should be distinguished from thrombi, cardiac tumour, myxomatous changes, strands or non-infective vegetations, as well as Lambl's excrescences. ${ }^{3)}$ Careful assessment is required to free us from the traps. Early and accurate diagnosis of IE is crucial to achieve the best therapeutic strategy. Surgery is superior to medical intervention in RIE because surgical intervention can remove all infected tissues adequately. ${ }^{13)}$ The whole treatment process of this case just confirmed the advantage of surgery.

\section{Disclosures}

Conflicts of interest: No potential conflicts of interest to disclose.

\section{Acknowledgments}

I thank Yan-Sha Chen and Jie Fang (Department of Clinical Laboratory) for supporting the work.

\section{References}

1. Chen J, Lu S, Hu K, et al. Clinical Characteristics and Surgical Treatment of Infective Endocarditis With Bicuspid Aortic Valve. Int Heart J 2017; 58: 220-4.

2. Baddour L, Wilson W, Bayer A, et al. Infective Endocarditis in Adults: Diagnosis, Antimicrobial Therapy, and Management of Complications: A Scientific Statement for Healthcare Professionals From the American Heart Association. Circulation 2015; 132: $1435-86$.

3. Xie J, Liu S, Yang J, Xu J, Zhu G. Inaccuracy of transthoracic echocardiography for the identification of right-sided vegetation in patients with no history of intravenous drug abuse or cardiac device insertion. J Int Med Res 2014; 42: 837-48.

4. Oylumlu M, Ercan S, Basanalan F, Davutoglu V. Both-sided native valve endocarditis in an intravenous drug misuser. BMJ Case Rep 2013; 2013: bcr2013201980.

5. Tyagi S, Patki S, Vaideeswar P, Meshram V. Both-sided native valve infective endocarditis in a drug addict with incidental pneumoconiosis. J Forensic Leg Med 2018; 58: 41-3.

6. Lee M, Chang S, Choi S, et al. Clinical features of right-sided infective endocarditis occurring in non-drug users. J Korean Med Sci 2014; 29: 776-81.

7. Birkenkamp K, Jin J, Shivashankar R, Jouni H, Baddour L, Blauwet L. Ventricular septal defect and bivalvular endocarditis. Avicenna J Med 2015; 5: 21-3.

8. Akinosoglou K, Apostolakis E, Marangos M, Pasvol G. Native valve right sided infective endocarditis. Eur J Intern Med 2013; 24: 510-9.

9. Fazili T, Riddell S, Kiska D, et al. Streptococcus anginosus Group Bacterial Infections. Am J Med Sci 2017; 354: 257-61.

10. Forster R, de Campos F, Lovisolo S, Aiello V, Martines J. Infective endocarditis with left to right intracardiac fistula due to Streptococcus anginosus - a rare complication caused by an even rarer bacterium. Autops Case Rep 2013; 3: 13-22.

11. Tran M, Caldwell-McMillan M, Khalife W, Young V. Streptococcus intermedius causing infective endocarditis and abscesses: a report of three cases and review of the literature. BMC Infect Dis 2008; 8: 154 .

12. Woo P, Tse H, Chan K, et al. "Streptococcus milleri" endocarditis caused by Streptococcus anginosus. Diagn Microbiol Infect Dis 2004; 48: 81-8.

13. Wang T, Oh T, Voss J, et al. Characteristics and outcomes for right heart endocarditis: six-year cohort study. Heart Lung Circ 2014; 23 : 625-7. 